\title{
Novedades florísticas y corológicas para la provincia de Córdoba (España)
}

\author{
Emilio Reyes Sepúlveda1, Gloria Martínez-Sagarra² \& Juan Antonio Devesa ${ }^{2 *}$ \\ ${ }^{1} \mathrm{C} /$ María la Judía 10, 2º-1D, 14011-Córdoba. \\ ${ }^{2}$ Departamento de Botánica, Ecología y Fisiología Vegetal. Edificio José Celestino Mutis, Campus de Rabanales, \\ Universidad de Córdoba, 14071 (Córdoba)
}

\section{Correspondencia}

J.A. Devesa

e-mail: bv1dealj@uco.es

Recibido: 4 septiembre 2017

Aceptado: 20 noviembre 2017

Publicado on-line: diciembre 2017

\section{Floristic and chorologic novelties for the Flora of Cordoba province (Spain)}

Palabras clave: Flora vascular, corología, Córdoba.

Key words: Vascular flora, chorology, Córdoba.
Se comentan 32 taxones de plantas vasculares cuya localización es de particular interés para la Flora de Córdoba. En muchos de los casos se amplía el conocimiento corológico del taxón en la provincia, como es el caso de muchos taxones basófilos que hasta ahora eran conocidos solo de la Campiña y Sierras Subbéticas, al sur del río Guadalquivir, y que han sido localizados en Sierra Morena, en la mitad septentrional del territorio, viviendo la mayor parte de ellos en los muy localizados enclaves calcáreos que aparecen aquí diseminados [v.gr., Roemeria hybrida (L.) DC.; Dorycnium pentaphyllum Scop.]. También, se dan a conocer nuevas recolecciones de taxones muy raros en la provincia (v.gr., Pilularia minuta Durieu), así como nuevas poblaciones que amplían significativamente el área de taxones hasta ahora muy localizados [v.gr., Quercus pyrenaica Willd.; Scrophularia oxyrrhincha Coincy; Linaria saxatilis (L.) Chaz.].

Se aportan también otros hallazgos que entrañan la novedad florística para este territorio [Galenia secunda (L. fil.) Sond.; Polygonum amphibium L.; Chenopodium chenopodioides Batt.; Alliaria petiolata (M. Bieb.) Cavara \& Grande; Barbarea intermedia Boreau; Lunaria annua L., Sp. PI., 653 (1753) subsp. annua; Galega cirujanoi García-Mur. \& Talavera; Lavandula viridis L'Her.; Sibthorpia europea L.; Klasea integrifolia (Vahl) Greuter; Fuirena pubescens (Poir.) Kunth; Scilla verna subsp. ramburii (Boiss.) K. Richt.], para Andalucía Occidental (Mollugo verticillata L.; Pilosella capillata (Arv.-Touv.) Mateo), o incluso para
Andalucía [Lindernia dubia (L.) Pennell]. Todos los pliegos-testimonio se encuentran depositados en el Herbario de la Universidad de Córdoba (COFC).

\section{PTERIDOPHYTA}

Pilularia minuta Durieu in Bory \& Durieu, Expl. Sci. Algérie, Atlas, PI. 38 figs. 1-20 (1848)

CÓRDOBA. Embalse Dehesa Boyal, término de Villanueva del Duque, 30STH2451, 580 m.s.n.m., 18-VI2011, E. Reyes (COFC 64349).

Especie nativa de la Región Mediterránea, que en la Península Ibérica se conoció inicialmente del Algarve (Paiva, 1986), y de algunas localidades dispersas por el territorio, como Zamora (Aedo et al., 1993, y otras localidades con posterioridad, vide anthos.es), Huelva (Sánchez Gullón, 1999), Sevilla (Hellmann \& Hellmann, 1993; Benítez et al., 2008), y también de las Islas Baleares (Menorca; Rita, 1987). Más recientemente ha sido dada a conocer de la provincia de Valladolid (Castro et al., 2007), León (Castro et al., 2009), y Cuenca (García Cardo, 2011).

También, se conoce de la provincia de Córdoba (Delgado et al., 2007), ocupando un espacio reducidísimo en la laguna de Peña Zorra, en el término municipal de La Granjuela (NW de la provincia). Se trata, sin duda, de una especie muy rara en el territorio peninsular, y por ello tipificada como vulnerable en las sucesivas listas rojas (VV. AA., 2000; Moreno, 2008) y Atlas de Flora Amenazada (Bañares et al., 2004). Por ello, el hallazgo de una nueva población en la provincia 
de Córdoba merece ser reseñada, esta vez en las inmediaciones de Villanueva del Duque, a 1,5 km del pueblo, formando poblaciones muy reducidas en el borde de un embalse, junto con Marsilea batardae Launert, Typha dominguensis Pers., Heliotropium supinum L., Glinus lotoides L., etc.

\section{DICOTYLEDONEAE}

Roemeria hybrida (L.) DC., Syst. Nat. 2, 92 (1821) Chelidonium hybridum L., Sp. PI., 506 (1753) CÓRDOBA. El Barrosillo, término de Fuente Obejuna, 30STH9037, 540 m.s.n.m., 3-V-2009, E. Reyes (COFC 64361).

Especie de la Región Mediterránea y el SW de Asia, con claras apetencias por los suelos básicos algo nitrificados, y que en el territorio provincial está bien representada en su mitad sur, tanto en la Campiña como en las Sierras Subbéticas (Pujadas, 1986; García-Montoya, 1995; Triano, 1998). En la mitad norte de la provincia, en el ámbito de Sierra Morena, donde hay un neto predominio de los materiales ácidos, se ha encontrado una población aislada, en un afloramiento básico en el término de Fuente Obejuna.

Galenia secunda (L. fil.) Sond. in Harv. \& Sond., Fl. Cap. 2, 474 (1862)

Aizoon secundun L. fil., Suppl. PI., 281 (1782)

CÓRDOBA. Embalse de Sierra Boyera, término municipal de Peñarroya-Pueblonuevo, 30SUH0039, 500 m.s.n.m., 24-VI-2012, E. Reyes (COFC 64353).

Neófito oriundo del sur de África, naturalizado en muchas partes del mundo (Castroviejo, 1990a). En la Península lbérica se conoce de las regiones costeras del $\mathrm{N}$ y SW de España, en particular de Asturias (Díaz \& Fernández, 1978) y de las provincias de Cádiz (Molesworth, 1976; Candau \& Pastor, 1986; Valdés et al., 2005a) y Huelva (Sánchez \& Rubio, 1999). Su presencia en las inmediaciones del embalse de Sierra Boyera constituye una novedad para la flora de la provincia de Córdoba, y extiende su área por Andalucía Occidental.

Quercus pyrenaica Willd., Sp. PI. 4, 451 (1805) CÓRDOBA. La Navilla, término municipal de Villanueva del Rey, 30SUH0926, 780 m.s.n.m., 18-XI2001, E. Reyes (COFC 64343); Cerro de la Venta, Sierra del Hoyo, término municipal de Belmez, 30SUH0136, 630 m.s.n.m., 28-X-2000, E. Reyes (COFC 64341).

El melojo o rebollo es un árbol endémico del NW de Marruecos, W y SW de Francia, y Península Ibérica (Amaral Franco, 1990), región esta última en la que su presencia es frecuente en la mitad septentrional, y escasa en la meridional (http,// www.anthos.es/), pues se localiza aquí solo en los sistemas montañosos con mayor precipitación, ya que se distribuye en áreas con precipitaciones anuales superiores a los $650 \mathrm{~mm}$, y su presencia en el piso mesomediterráneo está ligada a condiciones de elevada humedad ambiental y temperaturas frescas (García \& Jiménez, 2009).

Hasta la fecha, los bosques de melojo se conocían solo del noreste de la provincia (Rivas Goday \& Rivas Martínez, 1971), en el seno del Parque Natural de Cardeña-Montoro, restringidos a los términos municipales de Cardeña y Venta del Charco. El hallazgo de las poblaciones mencionadas extiende, por tanto, el área provincial de distribución de la especie a otras serranías septentrionales más occidentales, muy alejadas de las anteriores (unos $75 \mathrm{~km}$ ), culminadas por los cerros de Malpasillo (881 m.s.n.m.) y Maleto (878 m.s.n.m.), en las que el régimen de lluvias es superior al de los terrenos circundantes.

Mollugo verticillata L., Sp. PI., 89 (1753)

CÓRDOBA. Embalse de Sierra Boyera, término municipal de Peñarroya-Pueblonuevo, 30SUH0039, 500 m.s.n.m., 12-IX-2009, E. Reyes (COFC 64344).

Neófito originario de América tropical (Castroviejo, 1990b) y con escasa presencia en la flora peninsular, pues se conoce solo de las provincias de Cáceres (Belmonte, 1983; Amor et al., 1993) y Huelva (Sánchez \& Verloove, 2013), y de la Región de Tras-os-Montes en Portugal (Domingues de Almeida, 1999). El presente hallazgo en la provincia de Córdoba es novedoso para la flora de Andalucía Occidental.

Spergularia nicaeensis Sarato ex Burnat, FI. Alpes Marit. 1, 269 (1892)

CÓRDOBA. Mina del Porvenir de la Industria, término municipal de Fuente Obejuna, 30STH9642, 470 m.s.n.m., 23-V-2010, E. Reyes (COFC 64350).

Especie no muy frecuente en la provincia, conocida solo de algunos enclaves de la Campiña o inmediatos a la vega del Guadalquivir (Pujadas \& Hernández, 1987). Su hallazgo en el norte extiende su área de distribución a Sierra Morena, aunque de manera mucho más localizada de lo que lo está en la mitad meridional.

Silene laeta (Aiton) Godr. in Mém. Soc. Roy. Sci. Nancy 1846, 174 (1847)

Lychnis laeta Aiton, Hort. Kew. 2, 118 (1789)

CÓRDOBA. Los Villares, término municipal de Córdoba, 30SUH4002, 540 m.s.n.m., 30-IV-2015, E. Reyes (COFC 64352).

Especie del W de la Región Mediterránea, y el SW de Asia (Talavera, 1990), cuya área en la Península Ibérica se extiende por la mitad occidental (http,//www.anthos.es/). En la provincia 
de Córdoba solo se conocía del este de la comarca de Los Pedroches (Melendo \& Cano, 1997), ampliándose notablemente su área con el hallazgo descrito.

\section{Polygonum amphibium L., Sp. PI., 361 (1753)}

CORDOBA. Estación del Zújar, término municipal de Hinojosa del Duque, 30STH9671, 280 m.s.n.m., 6-VI2009, E. Reyes (COFC 64354). Finca de Madroñiz, márgenes del río Zújar, término municipal de El Viso, 30SUH1684, 355 m.s.n.m., 21-V-2017, J.A. Devesa \& G. Martínez-Sagarra (COFC 64327)

Especie de distribución casi subcosmopolita y que está muy extendida por buena parte de la Península Ibérica en aguas estancadas o a orillas de arroyos y ríos (Villar, 1990). Sin embargo, en Andalucía Occidental solo se conocía del litoral onubense (Castroviejo et al., 1980; Rivas Martínez et al., 1980; Pastor, 1987b), por lo que su presencia en el NW de la provincia de Córdoba amplía notablemente su área en este territorio y constituye la primera cita para la provincia de Córdoba.

Chenopodium chenopodioides Batt. in Batt. \& Trab., Fl. Algérie (Dicot.), 755 (1890)

CÓRDOBA. Cauce seco del río Zújar, finca de Madroñiz, entre Belalcázar y la Estación de Belalcázar, término municipal de El Viso, 30SUH1684, 360 m.s.n.m., 11-X-2016, J.A. Devesa \& S. Jurado (COFC 64331).

Especie cuya área de distribución se extiende por Europa, Asia, y África (Uotila, 1990), y que en la Península Ibérica aparece en el NW y, sobre todo, en su mitad oriental, y es muy escasa o incluso falta en el resto del territorio (http,//www.anthos. es/). En Andalucía Occidental solo se conoce del litoral onubense (Pastor, 1987a; Granado et al., 1988), por lo que la población detectada extiende considerablemente el área y constituye la primera cita para la flora de la provincia de Córdoba.

Rumex palustris Sm., FI. Brit. 1, 394 (1800) CÓRDOBA. Iznájar, entorno del pantano, 30SUG8424, 420 m.s.n.m, 20-XII-2016, J.A. Devesa \& G. Martínez-Sagarra (COFC 64332)

Especie extendida por Europa, W de Asia, y NW de África (López, 1990). Su área de distribución en la Península Ibérica, dispersa e irregular, se ha completado mucho en los últimos años, e incluye localidades portuguesas, del Alto y Baixo Alentejo, y españolas de las provincias de Salamanca, Madrid, Huesca, Lérida, Barcelona, Gerona, Tarragona, Valencia, Alicante, Sevilla, Jaén, y Córdoba (http,//www.anthos.es/). En esta última provincia la especie es muy rara, y hasta la fecha solo se conocía de la Campiña (Pastor, 1987c), concretamente de las inmediaciones de Palma del Río (Pujadas, 1986; Pujadas \& Hernández, 1987;
García et al., 1989). El nuevo hallazgo extiende su área provincial a las Sierras Subbéticas.

Alliaria petiolata (M. Bieb.) Cavara \& Grande in Bull. Orto Bot. Regia Univ. Napoli 3, 418 (1913) Arabis petiolata M. Bieb., FI. Taur.-Caucas. 2, 126 (1808)

CÓRDOBA. Arroyo de la Canaleja, término municipal de Pozoblanco, 30SUH3930, 480 m.s.n.m, 7-IV-2009, E. Reyes (COFC 64338).

Especie de amplia distribución a nivel mundial (Fernández \& Castroviejo, 1993), y que en la Península lbérica está bien representada en la mayor parte de su territorio, aunque falta o es más rara en el cuadrante sudoccidental (http,//www. anthos.es/). De hecho, en Andalucía Occidental se conoce solo de la Sierra de Aracena, Huelva (Rivera \& Cabezudo, 1985; Clemente \& Hernández, 1987; Diosdado et al., 1993), por lo que su hallazgo en Córdoba amplía su área y constituye la primera cita para la flora de esta provincia.

\section{Barbarea intermedia Boreau, Fl. Centre France 2, 48 (1840)}

CÓRDOBA. Urbanización de Las Siete Fincas, término municipal de Córdoba, 30SUH3800, 480 m.s.n.m., 22-IV-2010, E. Reyes (COFC 64345).

Nativa del W y SW de Europa (Fernandes, 1993), esta especie aparece bien representada en la mitad norte de la Península Ibérica, y solo de manera puntual y aislada en la mitad meridional (http,//www.anthos.es/). En Andalucía occidental solo se la conocía de la provincia de Huelva (Rivera \& Cabezudo, 1985; Sánchez \& Rubio, 2002; Sánchez \& Weickert, 2002), por lo que su presencia en Córdoba, no solo constituye una novedad florística sino que amplía notablemente su distribución en el territorio.

Lunaria annua L., Sp. Pl., 653 (1753) subsp.
annua

CÓRDOBA. Urbanización de Las Siete Fincas, término municipal de Córdoba, 30SUH3800, 460 m.s.n.m., 27-III-2009, E. Reyes (COFC 64349).

Especie del SE de Europa, a menudo utilizada en jardinería y con frecuencia escapada de cultivo y naturalizada (García Adá, 1993). Este es el caso de la población cordobesa, que constituye la primera cita del taxón para la flora de esta provincia.

Calicotome villosa (Poir.) Link in Neues J. Bot. 2, 51 (1808) (1789)

Spartium villosum Poir., Voy. Barbarie 2, 207

CÓRDOBA. Adamuz, El Puerto, 30SUH5713, 400 m.s.n.m., 7-X-2008, E. Reyes (COFC 64363).

Especie de la Región Mediterránea, que en la 
Península lbérica se localiza sobre todo en el sur, en áreas por lo general próximas al mar, aunque también penetra hacia el interior (García Murillo, 1999a), como sucede en la provincia de Sevilla (Morales et al., 1998) y de Córdoba. Aunque la especie no ha sido indicada en esta última provincia en el tratamiento para Flora iberica, ésta se conoce de las Sierras Subbéticas (Muñoz \& Domínguez, 1985), y ahora de Adamuz, cita que amplía el área del taxón en la provincia a Sierra Morena.

Galega cirujanoi García-Mur. \& Talavera in Anales Jard. Bot. Madrid 57, 218 (1999)

CÓRDOBA. Río Guadalbarbo, término municipal de Pozoblanco, 30SUH3427, 450 m.s.n.m., 22-V-2011, E. Reyes (COFC 64351); ibídem, 3-VII-2017, E. Reyes (COFC 64348).

Especie del E de la Región Mediterránea, y del W y SW de la Península Ibérica (García Murillo, 1999b), en donde hasta ahora era conocida solo del W de Portugal (Estremadura y Ribatejo; Talavera, 1999), y de las provincias españolas de Badajoz y Huelva (Talavera, 1999; Valdés et al., 2005b; Sánchez et al., 2010). Su hallazgo en el norte de Córdoba constituye una novedad florística para la provincia, y amplía el área conocida del taxón.

Dorycnium pentaphyllum Scop., FI. Carniol. ed. 2, 2, 87 (1771)

CÓRDOBA. Las Caleras, término municipal de Fuente Obejuna, 30STH9044, 580 m.s.n.m., 24-V-2014, E. Reyes (COFC 64362).

Especie del W de la Región Mediterránea, distribuida por casi toda la Península Ibérica (Díaz, 2000), si bien en la provincia de Córdoba se conocía solo del sur, de la Campiña y las Sierra Subbéticas (Muñoz \& Domínguez, 1985; GarcíaMontoya, 1995; Triano, 1998). Su hallazgo en el norte de la provincia extiende su presencia a Sierra Morena.

Datura innoxia Mill., Gard. Dict. ed. 8, n.o 5 (1768) CÓRDOBA. Córdoba, márgenes del río Guadalquivir, 7-X-2016, 34SUG4393, 92 m.s.n.m., J.A. Devesa \& P. Abad (COFC 62812).

Neófito originario de América Central (Gallego, 2012), que en la Península Ibérica aparece naturalizado sobre todo en las regiones litorales del este y sur de España, y de manera muy dispersa hacia el interior (http,//www.anthos.es/). En la provincia de Córdoba su presencia había sido indicada solo en Los Pedroches, en el término de Belalcázar (Jiménez \& Ruiz de Clavijo, 1990), pero se ha visto en otros lugares de Sierra Morena, y también en los márgenes del río Guadalquivir, a su paso por Córdoba, donde es relativamente abundante.
Lavandula viridis L'Her., Sert. Angl., 19, tab. 21 (1789)

CÓRDOBA. Finca La Baja, término municipal de Hornachuelos, 30STH9206, 400 m.s.n.m., 26-IV-2015, E. Reyes (COFC 64336).

Especie endémica de Macaronesia (Azores y Madeira) y el SW de la Península lbérica (Morales, 2010). En Andalucía Occidental se conocía solo del norte de las provincias de Huelva y Sevilla (Devesa, 1987), siendo muy destacable su hallazgo en la Sierra Morena cordobesa, en el ámbito del Parque Natural de Hornachuelos. Aparece aquí en matorrales bajo encinas y alcornoques, a menudo junto a su híbrido con $L$. stoechas subsp. luisieri (Rozeira) Rozeira. Se trata de la primera cita para la provincia de Córdoba.

Lindernia dubia (L.) Pennell in Monogr. Acad. Nat. Sci. Philadelphia 1, 141 (1935)

Gratiola dubia L., Sp. PI., 17 (1753)

CÓRDOBA. Fuente la Zarza, Hinojosa del Duque, márgenes de un embalse, 30SUH0457, 560 m.s.n.m., 5-VI-2016, E. Reyes (COFC 64359).

Neófito originario del $\mathrm{E}$ de Norteamérica, que aparece naturalizado de manera muy dispersa por mitad septentrional de la Península Ibérica (Rico, 2009a; http,//www.anthos.es/). Su hallazgo constituye una novedad para la flora de Córdoba, y para Andalucía.

Scrophularia oxyrrhincha Coincy in J. Bot. (Morot) 12(1), 4 (1898)

CÓRDOBA. Peñón de Peñaladrones, término municipal de Belmez, 30STH1540, 740 m.s.n.m., 9-IV2017, E. Reyes (COFC 64337).

Endemismo de Sierra Morena y del sur de los Montes de Toledo, cuya área se restringe a las provincias de Badajoz, Ciudad Real y Córdoba (Ortega Olivencia, 2009). En la provincia de Córdoba se conocía del límite septentrional de Los Pedroches, y de las Sierras de BelalcázarSanta Eufemia (http,//www.anthos.es/), siempre de manera muy localizada, en crestones y fisuras de materiales fundamentalmente cuarcíticos. Su hallazgo en el término de Belmez, unos $50 \mathrm{~km}$ más al sur y en poblaciones separadas de las anteriores por la penillanura granítica pedrocheña, es de particular importancia corológica, dada la poca frecuencia con que dicha especie se prodiga en el territorio.

Linaria saxatilis (L.) Chaz., Suppl. Dict. Jard. 2, 39 (1790)

Antirrhinum saxatile L., Cent. PI. I, 16 (1755)

CÓRDOBA. Peña Crispina, término de Espiel, 30SUH1836, 700 m.s.n.m., 5-05-2007, E. Reyes (COFC 64346). 
Endemismo de la Península Ibérica (Sáenz \& Bernal, 2009) que, al igual que el anterior, es frecuente en fisuras, rellanos y repisas de roquedos cuarcíticos, y que hasta la fecha se conocía solo de las Sierras de Belalcázar-Santa Eufemia (Ruiz de Clavijo \& Muñoz, 1985; Jiménez \& Ruiz de Clavijo, 1997). También aquí hay una extensión significativa de su área de distribución hacia el sur de Sierra Morena, a la misma distancia y complejo de sierras.

Sibthorpia europea L., Sp. PI., 631 (1753)

CÓRDOBA. Chimorra (término de Alcaracejos), 30SUH3132, 790 m, 24-V-2005, E. Reyes (COFC 64355).

Especie de la Región Macaronésica y del W de Europa, que alcanza algunos países del E de la Región Mediterránea y las montañas de África tropical (Rico, 2009b). En la Península Ibérica es frecuente en el norte y en la mitad occidental (http,// www.anthos.es/), pero en Andalucía Occidental solo se la conoce de la provincia de Cádiz (Pérez Lara, 1889; Willkomm, 1893; Rivas Goday, 1968; Molesworth, 1971; Díez et al., 1986; Valdés, 1987, y otros muchos). Su hallazgo en la Sierra Morena cordobesa constituye una novedad para la flora de esta provincia, y amplía su área de distribución en el territorio andaluz.

Xeranthemum inapertum (L.) Mill., Gard. Dict. ed. $8,{ }^{\circ}{ }^{\circ} 2(1768)$ (1753)

$X$. annuum var. inapertum L., Sp. PI., 858

CÓRDOBA. Sierra del Castillo, Espiel, 30SUH2126, 600 m.s.n.m., 14-V-2017, E. Reyes (COFC 64347).

Especie de la Región Mediterránea, SW y C de Europa, y SW de Asia, extendida por la mayor parte de la Península Ibérica, aunque rara en el oeste (http,//www.anthos.es/). En la provincia de Córdoba se conoce de la Campiña y de las Sierras Subbéticas (Muñoz \& Domínguez, 1985; García-Montoya, 1995; Triano, 1998), área que ha de ampliarse a los enclaves calcáreos de Sierra Morena.

Klasea integrifolia (Vahl) Greuter in Willdenowia 35, 235 (2005)

Cynara integrifoliaVahl, Symb. Bot. 1, 68 (1790) CÓRDOBA. Loma de Buenavista, término municipal de Pozoblanco, 30SUH3630, 780 m.s.n.m., 6-VI-2011, E. Reyes (COFC 64358).

Endemismo de la Península Ibérica distribuido, fundamentalmente, por el centro y el cuadrante sudoccidental, pero del que no existen testimonios previos de su presencia en la provincia de Córdoba (Cantó, 2014), si bien la imagen de esta especie publicada en Devesa (2016), corresponde a plantas de esta población cordobesa.

Centaurea depressa M. Bieb., Fl. Taur.-Caucas. 2, 346 (1808)

CÓRDOBA. El Barrosillo, término de Fuente Obejuna, 30STH 9037, 570 m.s.n.m., 1-V-2016, E. Reyes (COFC 64357).

Neófito oriundo del centro y sudoeste de Asia, hoy día naturalizado en muchas regiones del mundo, entre ellas la Península lbérica, en donde aparece sobre todo en la mitad oriental (MedinaGavilán, 2015). En Andalucía Occidental se conoce solo de Sevilla (Medina-Gavilán, 2015) y Córdoba (Devesa et al., 2014), en este último caso de las Sierras Subbéticas. Su hallazgo en Sierra Morena amplía notablemente su área en la provincia.

Pilosella capillata (Arv.-Touv.) Mateo, Cat. FI. Prov. Teruel, 141 (1990)

Hieracium capillatum Arv.-Touv., Hier. Gall. Hisp. Cat., 7 (1913)

CÓRDOBA. Subida a la Tiñosa, Sierra de Horconera, 30SUG8938, 1360 m.s.n.m., 9-VI-2017, J.A. Devesa, I. Hidalgo \& G. Martínez-Sagarra (COFC 64326).

Es la primera vez que se indica con testimonio la presencia de una especie del género Pilosella en la provincia de Córdoba, cita que implica el reconocimiento del género en la Flora Vascular de Andalucía Occidental, donde no había sido contemplado (Valdés et al., 1987).

\section{MONOCOTYLEDONEAE}

Damasonium polyspermum Coss., Notes PI. Crit., 47 (1849)

CÓRDOBA. Las Caleras, término municipal de Fuente Obejuna, 30STH 9143, 545 m.s.n.m., 6-VI-2010, E. Reyes (COFC 64360).

Especie del W de la Región Mediterránea (Talavera \& Talavera, 2010) y dispersa por la Península Ibérica (http,//www.anthos.es/). Recientemente fue indicada para la flora de Córdoba (Muñoz \& Díaz, 2016), en concreto de la laguna de Casasola, en las inmediaciones de la población de Baena (SE de la provincia). El nuevo hallazgo amplía su área en el territorio a Sierra Morena.

Fuirena pubescens (Poir.) Kunth, Enum. PI. 2, $182(1837)$

Carex pubescens Poir., Voy. Barbarie 2, 254 (1789)

CÓRDOBA. Ladera SE de Chimorra, término municipal de Alcaracejos, 30SUH3430, 600 m.s.n.m., 29-X-2010, E. Reyes (COFC 64356).

Especie distribuida por la Región Mediterránea, SW de Asia y regiones tropicales del mundo 
(Castroviejo, 2007), que en la Península Ibérica aparece de manera dispersa en el cuadrante suroccidental (http,//www.anthos.es/). De hecho, en Andalucía Occidental su presencia se conocía solo de las áreas costeras de las provincias de Cádiz y Huelva (Tutin, 1975; Rivas Martínez et al., 1980; Gil et al., 1985; Silvestre, 1987; Pérez Latorre et al., 1999, etc.), por lo que esta cita de Sierra Morena, nueva para la provincia de Córdoba, extiende considerablemente su área hacia el interior.

Stipa apertifolia Martinosvky in Preslia 39, 274 (1967)

Stipa dasyvagynata Martinosvky in Anal. Inst.

Bot. Cavanilles 27, 61 (1970)

CÓRDOBA. Subida a la Tiñosa, Sierra de Horconera, 30SUG8938, 1360 m.s.n.m., 9-VI-2017, J.A. Devesa, I. Hidalgo \& G. Martínez-Sagarra (COFC 64330).

Se confirma la presencia del taxón, ya indicada por Triano (1998) aunque con reservas sobre su identificación.

Allium nigrum L., Sp. Pl. ed. 2, 430 (1762) [nom. cons.]

CÓRDOBA. Sierra del Castillo, Espiel, 30SUH2127, 580 m.s.n.m., 15-V-2010, E. Reyes (COFC 64340).

Especie de la Región Mediterránea, Macaronesia y el C de Europa (Aedo, 2013), que en la Península Ibérica aparece dispersa por el este y la mitad meridional (http,//www.anthos.es/). En la provincia de Córdoba se conocía solo de la Campiña (López et al., 2015) y de las Sierras Subbéticas (Muñoz \& Domínguez, 1985; García Montoya, 1995; Triano, 1998). Su hallazgo en el enclave calizo de la Sierra del Castillo extiende su área provincial a Sierra Morena.

\section{Scilla verna Huds.}

subsp. ramburii (Boiss.) K. Richt., PI. Eur. 1, 220 (1890)

S. ramburii Boiss., Elench. PI. Nov., 86 (1838)

CÓRDOBA. Entre Venta del Charco y Cardeña, finca Yegüerizo, 30SUH8532, 770 m.s.n.m., Devesa \& Martínez-Sagarra (64329).

Endemismo del W, C y $\mathrm{S}$ de la Península Ibérica, y el NW de África (Marruecos) (Almeida \& Crespi, 2013), dado a conocer en Andalucía Occidental por Willkomm (1862), y que al parecer se restringe aquí a las áreas litorales de la provincia de Huelva y Cádiz (Pau, 1895; Gil et al., 1985; Díez \& Pastor, 1985; Pastor, 1987d; Sánchez-Gullón, 1999; Valdés et al., 2006; López-Albacete et al., 2007, entre otras referencias). Su hallazgo en Los Pedroches constituye la primera cita para la flora cordobesa, y amplía notablemente su área en el territorio.
Epipactis microphylla (Ehrh.) Sw. in Kongl. Vetensk. Acad. Nya Handl. 21, 232 (1800)

Serapias microphylla Ehrh., Beitr. Naturk. 4, 42 (1789)

CÓRDOBA. Arroyo Bejarano, término municipal de Córdoba, 30SUH3501, 400 m.s.n.m., 30-V-2014, E. Reyes (COFC 64342).

Especie distribuida por Europa y el W de Asia, que en la Península Ibérica se extiende por el sur y la mitad oriental (Crespo, 2005). En la provincia de Córdoba se conocía solo de las Sierras Subbéticas (Muñoz \& Domínguez, 1985), por lo que el nuevo hallazgo extiende su presencia hasta el sur de Sierra Morena.

\section{Agradecimientos}

A Enrique Sánchez Gullón por sus observaciones sobre Barbarea intermedia.

\section{Referencias}

Aedo, C. (2013). Allium. In, E. Rico et al. (eds.) Flora iberica, 20, 220-273. CSIC. Madrid.

Aedo, C., Aldasoro, J.J., Argüelles, J.M., Díaz Alonso, J.L. González Del Valle, J.M., Herrá, C., Laínz, M., Moreno Moral G., Patallo, J. \& Sánchez Pedraja, Ó. (1993). Contribuciones al conocimiento de la flora cantábrica. Fontqueria, 36, 349-374.

Almeida Da Silva, R. M. \& Crespi, A. L. (2013). Scilla. In, E. Rico et al. (eds.) Flora iberica, 20, 145-156. CSIC. Madrid.

Amaral Franco, J. Do (1990). Quercus. In, S. Castroviejo, M. Laínz et al. (eds.) Flora iberica, 2, 15-36. CSIC. Madrid.

Amor, A., Ladero, M. \& Valle, C. J. (1993). Flora y vegetación vascular de la comarca de la Vera y laderas meridionales de la Sierra de Tormantos (Cáceres, España). Stud. Bot., 11, 11-207.

Bañares, Á., Blanca, G., Güemes, J., Moreno, J. C. \&. Ortiz, S., eds. (2004). Atlas y Libro Rojo de la Flora Vascular Amenazada de España. Dirección General de Conservación de la Naturaleza. Madrid, 1.069 pp.

Belmonte, D. (1983). Datos florísticos sobre la comarca de las Corchuelas (Parque Natural de Monfragüe, Cáceres, España). III. Lazaroa, 5, 315-317.

Benítez Cruz, G., Delgado Vázquez, A.J. \& Rendón Vega, J. (2008). Notas pteridológicas. 54. Nuevas citas de Pilularia minuta Durieu (Marsileaceae) en Sevilla, Andalucía Occidental. Acta Bot. Malacitana, 33, 351-352.

Candau, P. \& Pastor, J. (1986). Contribución al conocimiento del polen de Aizoaceae en Andalucía Occidental. Lagascalia, 14, 189-195.

Cantó, P. (2014). Klasea. In, J.A. Devesa et al. (eds.) Flora iberica, 16(1), 243-263. CSIC. Madrid.

Castro, V., Llamas, F. \& Alonso, R. (2007). De Plantis Legionensibus. Notula XXII. Lazaroa, 28, 123-128.

Castro, V., Alonso, R. \& Llamas, F. (2009). De Plantis Legionensibus. Notula XXV. Lagascalia, 29, 322-329. 
Castroviejo, S. (1990a). Galenia. In, S. Castroviejo, M. Laínz et al. (eds.) Flora iberica, 2, 76. CSIC. Madrid.

Castroviejo, S. (1990b). Mollugo. In, S. Castroviejo, M. Laínz et al. (eds.) Flora iberica, 2, 93-95. CSIC. Madrid.

Castroviejo, S. (2007). Fuirena. In, S. Castroviejo et al. (eds.) Flora iberica, 18, 92-94. CSIC. Madrid.

Castroviejo, S., Valdés Bermejo, E., Rivas-Martínez, S. \& Costa, M. -1980). Novedades florísticas de Doñana. Anales Jard. Bot. Madrid, 36, 203-244.

Clemente, M. \& Hernández, J. E. (1987). Alliaria. In, B. Valdés, S. Talavera \& E.F. Galiano (eds.) Flora Vascular de Andalucía Occidental, 1, 380. Ed. Ketres. Barcelona.

Crespo, M.B. (2005). Epipactis. In, C. Aedo \& A. Herrero (eds.) Flora iberica, 21, 22-54. CSIC. Madrid.

Delgado, A.J., Jiménez Sánchez, M.L. \& Medina, L. (2007). Notas pteridológicas. 52. Pilularia minuta Durieu, a new fern for Córdoba province (Andalusia, Spain). Acta Bot. Malacitana 32, 247-248.

Devesa, J.A. (1987). Lavandula. In, B. Valdés, S. Talavera \& E.F. Galiano (eds.) Flora Vascular de Andalucía Occidental, 2, 450-455. Ed. Ketres. Barcelona.

Devesa, J.A. (2016). Las especies de Centaurea de la Península Ibérica y Baleares, 450 págs. Ucopress, Universidad de Córdoba. Córdoba.

Devesa, J.A., López Nieto, E., Arnelas, I., Blanca, G., Suárez-Santiago, V. N., Rodríguez, V. \& Muñoz, A. (2014). Centaurea. In, J.A. Devesa et al. (eds.) Flora iberica, 16(1), 342-603. CSIC. Madrid.

Díaz González, T.E. \& Fernández Prieto, J.A. (1978). Notas sobre la flora astur-leonesa. Rev. Fac. Ci. Univ. Oviedo, 17-19, 303-308.

Díaz Lifante, Z. (2000). Dorycnium. In, S. Talavera et al. (eds.) Flora iberica, 7(2), 812-823. CSIC. Madrid.

Díez Dapena, M. J. \& Pastor, J. (1985). Contribución al estudio del polen y semillas de la tribu Scilleae (Liliaceae) en Andalucía Occidental. Anales Jard. Bot. Madrid, 41(2), 351-360.

Díez Garretas, B., Cuenca, J. \& Asensi, A. (1986). Datos sobre la vegetación del subsector aljíbico (provincia Gaditano Onubo Algarviense). Lazaroa ,9, 315-332.

Diosdado, J.C., Santa-Bárbara, C., Vioque, J., Juan, R. \& Pastor, J. (1993). Números cromosómicos para la Flora Española, 691-719. Lagascalia, 17, 173-184.

Domingues de Almeida, J. (1999). Adiciones corológicas a Flora Iberica I-VIII (1986-1998). Anales Jard. Bot. Madrid, 57(2), 431-433.

Fernandes, R.B. (1993). Barbarea. In, S. Castroviejo, M. Laínz et al. (eds.) Flora iberica, 4, 98-101. CSIC. Madrid.

Fernandez Arias, M. I. \& Castroviejo, S. (1993). Alliaria. In, S. Castroviejo, M. Laínz et al. (eds.) Flora iberica, 4, 38. CSIC. Madrid.

Gallego, M. J. (2012). Datura. In, S. Talavera et al. (eds.) Flora iberica 11, 216-224. CSIC. Madrid.

García Adá, R. (1993). Lunaria. In, S. Castroviejo, M. Laínz et al. (eds.) Flora iberica, 4, 165-167. CSIC. Madrid.

García-Montoya, F. (1995). Flora de la Campiña Alta cordobesa. Ed. Subbética de Impresiones. Cabra.

García Murillo, P. (1999a). Calicotome. In, S. Talavera et al. (eds.) Flora iberica, 7(1), 182-188. CSIC. Madrid.

García Murillo, P. (1999b). Galega. In, S. Talavera et al. (eds.) Flora iberica, 7(1), 267-274. CSIC. Madrid.

García Cardo, O. (2011). Pilularia minuta Durieu en la Manchuela conquense. Fl. Montiber., 48, 7-11.

García, C., Pastor, J. \& Luque, T. (1989). Contribución al estudio cariológico del género Rumex (Polygonaceae). Acta Bot. Malacitana, 14, 129-140.

García, I. \& Jiménez, P. (2009). 9230 Robledales de Quercus pyrenaica y robledales de Quercus robur y Quercus pyrenaica del Noroeste ibérico. In, VV.AA., Bases ecológicas preliminares para la conservación de los tipos de hábitat de interés comunitario en España. Madrid, Ministerio de Medio Ambiente, y Medio Rural y Marino. $66 \mathrm{p}$.

Gil, J.M., Arroyo, J. \& Devesa, J.A. (1985). Contribución al conocimiento florístico de las sierras de Algeciras (Cádiz, España). Acta Bot. Malacitana ,10, 97-122

Granado, C., Luque, T. \& Pastor, J. (1988). Números cromosómicos para la Flora Española. 551-555. Lagascalia, 15, 133-136.

Hellmann. R \& Hellmann, V. (1993). Pilularia minuta Durieu (Marsileaceae) and Littorella uniflora (L.) Ascherson (Plantaginaceae) in South West Andalusia. Lagascalia, 17(1), 189-190.

Jiménez, M. \& Ruiz De Clavijo, E. (1990). Notas Taxonómicas y Corológicas para la Flora Vascular de Andalucía y del Rif. 16. Nuevas áreas para la flora de Andalucía Occidental. Lagascalia, 16(1), 132-145.

Jiménez, M. \& Ruiz De Clavijo, E. (1997). Catálogo florístico de la cuenca hidrográfica del río Zújar en la provincia de Córdoba. 89 pp. Monogr. Jardín Botánico de Córdoba, vol.6. Córdoba.

López González, G. (1990). Rumex. In, S. Castroviejo, M. Laínz et al. (eds.) Flora iberica, 2, 595-634. CSIC. Madrid.

López-Albacete, I., De Las Heras, M.A., Sánchez Gullón, E., Hidalgo, P.J. \& Muñoz Rodríguez, A.F. (2007). Aportaciones florísticas para Doñana. Lagascalia, 27, 390-402.

Medina-Gavilán, J. L. (2015). Contribuciones a la flora vascular de Andalucía (España). 189. Nueva cita de Centaurea depressa M. Bieb. (Asteraceae) para Andalucía Occidental. Acta Bot. Malacitana, 40, 249250.

Melendo, M. \& Cano, E. (1997). La clase IsoetoNanojuncetea en el Noreste de la Provincia de Córdoba (Sierra Morena, España). Monogr. Fl. Veg. Bética, 10, 127-142.

Molesworth Allen, B. (1971). Notas sobre helechos españoles. Lagascalia, 1, 83-87.

Molesworth Allen, B. (1976). Notas sobre algunas plantas de la provincia de Cádiz. Lagascalia ,6, 239-242.

Morales, R. (2010). Lavandula. In, R. Morales et al. (eds.) Flora iberica ,12, 484-496. CSIC. Madrid.

Morales, M., Delgado, J. M., Torrecilla, A., Tamajón, R. \& Muñoz, J. M. (1998). Notas taxonómicas y corológicas para la flora vascular de Andalucía y del Rif. 57. Novedades corológicas para la comarca de Sierra Norte en la provincia de Sevilla. Lagascalia, 20, 316331.

Moreno, J. C., coord. (2008). Lista Roja 2008 
de la flora vascular española. Dirección General de Medio Natural y Política Forestal (Ministerio de Medio Ambiente, y Medio Rural y Marino, y Sociedad Española de Biología de la Conservación de Plantas), Madrid, 86 pp.

Muñoz, J. \& Domínguez, E. (1985). Catálogo florístico del sur de la provincia de Córdoba. Ed. Tipografía Católica. Córdoba.

Muñoz Álvarez, J. M. \& Díaz Iglesias, M.L. (2016). Contribuciones a la flora vascular de Andalucía (España). 189. 203. Aportaciones a la flora vascular de Córdoba y Andalucía Occidental (España). Acta Bot. Malacitana, 41, 312-316.

Ortega Olivencia, A. (2009). Scrophularia. In, C. Benedí et al. (eds.) Flora iberica, 13, 97-134. CSIC. Madrid.

Paiva, J. (1986). Pilularia. In, S. Castroviejo, M. Laínz et al. (eds.) Flora iberica, 1, 69-71. CSIC. Madrid.

Pastor, J. (1987a). Chenopodium. In, B. Valdés, S. Talavera \& E.F. Galiano (eds.) Flora Vascular de Andalucía Occidental, 1, 171-177. Ed. Ketres. Barcelona.

Pastor, J. (1987b). Polygonum. In, B. Valdés, S. Talavera \& E.F. Galiano (eds.) Flora Vascular de Andalucía Occidental, 1, 277-283. Ed. Ketres. Barcelona.

Pastor, J. (1987c). Rumex. In, B. Valdés, S. Talavera \& E.F. Galiano (eds.) Flora Vascular de Andalucía Occidental, 1, 285-293. Ed. Ketres. Barcelona.

Pastor, J. (1987d). Scilla. In, B. Valdés, S. Talavera \& E.F. Galiano (eds.) Flora Vascular de Andalucía Occidental, 3, 440-443. Ed. Ketres. Barcelona.

Pau, C. (1895). Plantas de La Bética. Actas Soc. Esp. Hist. Nat., 24(2), 130-142

Pérez Lara, J. M. (1889). Florula gaditana. Pars tertia. Anales Soc. Esp. Hist. Nat., 18(1), 35-143.

Pérez Latorre, A.V., Galán De Mera, A., Navas, P., Navas, D., Gil, Y. \& Cabezudo, B. (1999). Datos sobre la flora y vegetación del Parque Natural de Los Alcornocales (Cádiz-Málaga, España). Acta Bot. Malacitana, 24, 133-184.

Pujadas, A. (1986). Flora arvense y ruderal de la Provincia de Córdoba. Edit. Universidad de Córdoba, Escuela Técnica Superior de Ingenieros Agrónomos.

Pujadas, A. \& Hernández Bermejo, J. E. (1987). Plantas Arvenses y Ruderales interesantes de la provincia de Córdoba. Acta Bot. Malacitana ,12, 231-236.

Rico, E. (2009a). Lindernia. In, C. Benedí et al. (eds.) Flora iberica, 13, 331-334. CSIC. Madrid.

Rico, E. (2009b). Sibthorpia. In, C. Benedí et al. (eds.) Flora iberica, 13, 434-437. CSIC. Madrid.

Rita, J. (1987). Notas Pteridológicas. 9. Pilularia minuta Durieu (Marsilaceae) en las Islas Baleares. Acta Bot. Malacitana, 12, 249-252.

Rivas Goday, S. (1968). Algunas novedades fitosociológicas de España Meridional. Collect. Bot. (Barcelona), 7, 997-1031.

Rivas Goday, S. \& Rivas Martínez, S. (1971). Vegetación. In, F. González García -Director- Estudio agrobiológico de la provincia de Córdoba, 243-262. CSIC. Madrid

Rivas Martínez, S., Costa, M., Castroviejo, S. \& Valdés, E. (1980). Vegetación de Doñana (Huelva, España). Lazaroa, 2, 5-189.

Rivera, J. \& Cabezudo, B. (1985). Aportaciones al conocimiento florístico de la Sierra de Aracena (Huelva, España). Acta Bot. Malacitana, 10, 61-78.

Ruiz De Clavijo, E. \& Muñoz, J. (1985). Algunas plantas interesantes de la provincia de Córdoba. Acta Bot. Malacitana, 10, 79-84.

Sáenz, L. \& Bernal, M. (2009). Linaria. In, C. Benedí et al. (eds.) Flora iberica, 13, 232-324. CSIC. Madrid.

Sánchez Gullón, E. (1999). Contribuciones a la flora vascular de Andalucía (España) y del Rif (Marruecos). 65. Novedades corológicas para la provincia de Huelva. Acta Bot. Malacitana, 24, 242-247.

Sánchez Gullón, E. \& Rubio García, J.C. (1999). Novedades florísticas para el litoral de Huelva y provincia. I. Lagascalia, 21, 7-16.

Sánchez Gullón, E. \& Rubio García, J.C. (2002). Novedades florísticas para el litoral de Huelva y provincia. II. Lagascalia, 22, 7-19.

Sánchez Gullón, E. \& Weickert, P. (2002). Plantas del herbario de D. Bruno Weickert herborizadas en la provincia de Huelva entre los años 1950/1974. Lagascalia, 22, 172-179.

Sánchez Gullón, E. \& Verloove, F. (2013). New records of interesting vascular plants (mainly xenophytes) in the Iberian Peninsula. IV. Folia Bot. Extremadurensis, 7, 29-34.

Sánchez Gullón, E., Fernández-Illescas, F., De Las Heras, M. A., Jiménez Nieva, F. J., Rodríguez Rubio, P. \& Muñoz Rodríguez, A. F. (2010). Novedades florísticas para el litoral de Huelva y provincia. III. Lagascalia, 30, 461-472.

Silvestre, S. (1987). Fuirena. In, B. Valdés, S. Talavera \& E.F. Galiano (eds.) Flora Vascular de Andalucía Occidental, 3, 236. Ed. Ketres. Barcelona.

Talavera, S. (1990). Silene. In, S. Castroviejo, M. Laínz et al. (eds.) Flora iberica, 2, 313-406. CSIC. Madrid.

Talavera, S. (1999). Sobre el tratamiento de la Tribu Austragaleale Bercht. \& J. Presl (Papilioideae, Leguminosae) en "Flora Ibérica". Anales Jard. Bot. Madrid ,57(1), 218-220.

Talavera, S. \& Talavera, M.M. (2010). Damasonium. In, S. Talavera et al. (eds.) Flora iberica, 17, 18-22. CSIC. Madrid.

Triano, E. (1998). Flora del Subbético cordobés. Catálogo, recursos y curiosidades. Ayuntamiento de Rute. Diputación provincial de Córdoba.

Tutin, T. G. (1975). Molinia in SW Spain. Lagascalia, 5, 73-75.

Uotila, P. (1990). Chenopodium. In, Castroviejo, S., Laínz, M., López Gonzáles, G., Montserrat, P., Muñoz Garmendia, F., Paiva, J. \& Villar, L. (eds.) Flora iberica 2,484-500. CSIC. Madrid.

Valdés, B. (1987). Sibthorpia. In, B. Valdés, S. Talavera \& E.F. Galiano (eds.) Flora Vascular de Andalucía Occidental, 2, 530. Ed. Ketres. Barcelona.

Valdés, B., Talavera, S. \& Galiano, E.F., eds. (1987). Flora Vascular de Andalucía Occidental, 3 vol. Ed. Ketres. Barcelona.

Valdés, B., Carmona, I., Girón, V. \& García, M.C. (2005aNotas taxonómicas y corológicas para la Flora de la Península Ibérica y El Maghreb. 93. Notas sobre la Flora de Doñana. 2. Algunas plantas del Parque Natural de Doñana nuevas para el Litoral de Cádiz. 
Lagascalia, 25, 187-192

Valdés, B., Santa Bárbara, C., Vicent, C. \& García García, M.C. (2005b). Notas taxonómicas y corológicas para la Flora de la Península Ibérica y El Maghreb. 98. Novedades corológicas para el Andévalo y la Sierra de Aracena (Provincia de Huelva, SO de España). Lagascalia, 25, 211-217.

Valdés, B., Carmona, I., Girón, V. \& Sánchez Gullón, E. (2006). Notas taxonómicas y corológicas para la Flora de la Península Ibérica y El Magreb. 119. Notas sobre la Flora de Doñana. 5. Nuevas áreas para el entorno de Doñana. Lagascalia, 26, 228-235.

VV. AA. (2000). Lista Roja de Flora Vascular Española (valoración según categorías UICN). Conservación Vegetal 6 (extra), 11-38.

Willkomm, H.M. (1862). Scilla. In, H.M. Willkomm \& J.M.C. Lange -eds.- Prodromus Florae hispanicae 1, 213-215. Stuttgart.

Willkomm, H.M. (1893). Supplementum Prodromi Florae Hispanicae... Stuttgart. 